\title{
Enhancement of the QoS Parameter of LEACH Protocol using Modified K -Means Algorithm
}

\author{
Arvinda kushwaha, Mohd Amjad
}

\begin{abstract}
Wireless Sensor Network (WSN) is a collection of battery operated sensors deployed in the monitoring area. A massive quantity of energy of the nodes is used in the internal and external communications. There is a need of energy saving mechanism for effective and efficient communication. In this paper, we propose a new Modified-LEACH (MD-LEACH) protocol for enhancing the Quality of Services $(Q o S)$ parameters. This hierarchical routing protocol (MD-LEACH) is inspired by the $k$-means clustering technique to consolidate sensor networks into clusters and acquire an enhanced QoS parameter. The kmeans algorithm efforts to improve the clustering procedure of LEACH protocol using Euclidean distance and prolong the lifespan of the sensor network. This algorithm forms the optimized clusters by a distance of cluster head from cluster nodes and energy of the nodes for designated the cluster heads. To evaluate the performance of the proposed approach, we used NS-2 simulator, and consider the QoS parameter, namely: packet delivery ratio, energy consumption, bandwidth, and throughput. The simulations results show that the MD-LEACH algorithm outperforms then the LEACH protocol by optimizing all QoS parameters and improved network performance.
\end{abstract}

Index Terms: cluster head-means algorithm, LEACH, MDLEACH, QoS, and WSN.

\section{INTRODUCTION}

Over the previous few eras, there has been a significant evolution in academia as well as the industry in the arena of WSNs due to the advancement in the sensor's abilities like sensing power, computation, and communication capabilities. WSNs possess different characteristics like power consumption constraints for nodes, ability to cope with node failures, heterogeneity and homogeneity of nodes, scalability to large scale of deployment, ability to withstand harsh environmental conditions, ease of use, and cross-layer design In WSN, various sensors are spread in the target area for monitoring and recording the physical terms of the environment that have small size, low processing power, less storage, and low energy abilities. These sensor nodes can sense the target and make an infrastructure-less wireless communication among them and base station (BS). Sensors accumulate the data from the target area and forward it to the BS directly or with the assistance of other sensors. The BS is connected to the server or other sensor networks with the help of wired/wireless links. Thus, the information is further sent to the end user via the internet. The BS is supposed to be reliable and is capable of performing any operation. WSNs are being used in different applications like battlefield surveillance, health care monitoring, forest fire detection, landslide detection, water quality monitoring, natural disaster prevention, structural health monitoring, data center monitoring and data logging, environmental conditions such as temperature, sound, pollution levels, humidity, wind, etc., industrial and consumer applications like machine health monitoring, industrial process monitoring and control etc., and so on. On the bases of above-discussed applications, we can categories the wireless sensor node deployment into two groups like deterministic and non-deterministic. In deterministic implementations, sensor nodes are placed in a controlled manner or manually at the selected locations where the deployment area is physically accessible such as city sense monitoring, soil monitoring, etc.

On the other hand, in non-deterministic deployments sensor nodes are deployed into physically inaccessible areas using other sources like sensors are dropped from an aircraft, e.g., battlefield surveillance and landslide detection, etc. The nondeterministic deployment is also called as random deployment. Now, we discuss the very first protocol called LEACH which we consider baseline protocol. The first hierarchical routing protocol is LEACH [1]. It enhances the QoS parameter like reduced energy consumption and optimizes delay, packet delivery ratio, bandwidth, and throughput etc. It is used the theory of data accumulation in which network is divided into a different group or clustered. Data aggregation is used for enhanced performance in terms of a lifespan of the system. There is a substantial similarity between the communication architecture of the cellular network and LEACH. The mobile network is a collection of cells where cells are called group or cluster. It is established on the signal amplitude, and a router is work as a cell header node or cluster- head. There is a specific cluster-head $(\mathrm{CH})$ election method that is based on probability function, and it has many criteria that are based on the preferred percentage of Cluster heads and the many times for the duration of which a node acquired the role of $\mathrm{CH}$. In this algorithm, there is a predetermined time interval which term as a round. Where every round has two phases first one is an initialization phase and the second one is a transmission phase.
Revised Manuscript Received on July 22, 2019.

Arvinda kushwaha, Department of computer Engineering, Jamia Millia Islamia, New Delhi, India.

Mohd. Amjad, Department of computer Engineering, Jamia Millia Islamia, New Delhi, India. 
The research community is following the clustering of sensor nodes to accomplish the aim of scalability (scalability) of the network. The clustering techniques can be divided into two parts: hierarchical clustering and clustering partitioned.

The method of clustering partitioned is more well-organized and faster than the hierarchical classification and consortium is partitioned based on solid suppositions in [2]. In the perspective of clustering of manuscript mostly used partition technique is the k-means algorithm that's why we used kmeans algorithm while there are so many partitioned techniques available. In this paper, we propose a new Modified-LEACH (MD-LEACH) protocol for enhancing the Quality of Services (QoS) parameters. This hierarchical routing protocol (MD-LEACH) is inspired by the k-means clustering technique to consolidate sensor networks into clusters and acquire an enhanced QoS parameter. The kmeans algorithm efforts to improve the clustering procedure of LEACH protocol using Euclidean distance and prolong the lifespan of the sensor network. This algorithm forms the optimized clusters by a distance of cluster head from cluster nodes and energy of the nodes for designated the cluster heads.

The remaining paper is systematized as follows. Section 2 presents associated work and details about k- means algorithms, Section 3 explain the proposed algorithm; Section 4 simulation results of the proposed algorithm and Section 5 present a conclusion that summarizes this work.

\section{RELATED WORK}

Recently many approaches are available for clustering of the sensor network to enhance the QoS of the sensor network. The classification method based on k-means has been presented which is surveyed below.

In $[3,4]$ authors presented "a protocol that is a grouping of two practices k-means algorithm and Minimum Transmission Energy (MTE). The resulted practice has distance as a condition for choosing the nodes entitled to arrangement the cluster". In $[5,6]$ et al. presented a "balanced k-means protocol that increases the lifespan of the network over LEACH, LEACH-C, and LEACH K-Means. This methodology was founded on the space equivalent distribution and energy equivalent distribution nodes". In [7, 8] authors presented a better technique of $\mathrm{CH}$ selection based on minimum Euclidean distance to form the clusters and to find efficient cluster head. In [9, 4] authors proposed an enhanced LEACH protocol that is based on the Elbow process is used to form the cluster and find to an optimal number of " $\mathrm{k}$ " by using the k-means algorithm. In [11, 1416] authors presented distributed Clustering techniques and in $[11,17-19]$ authors presented centralized clustering technique. In [11, 14-16] authors presented $\mathrm{CH}$ selection at the individual level or in $[20,21]$ authors discussed a technique by using neighbor coordination. In [22-25] authors discussed different clustering techniques in wireless sensor networks. In [11] authors proposed $\mathrm{CH}$ selection on the random basis and in [17-21] authors consider some predefined parameters such as residual energy for $\mathrm{CH}$ selection, inter-cluster communication cost [20], distance from the sink [19], the number of neighbor nodes (node degree) [16], and timer-based approach [19] etc.

While in centralized clustering, the Base station (BS) takings the full accountability of cluster creation. In this method, every node shares their all information like location, energy, and node ID to the Base station. The Base station decides the optimum clusters after evaluating the gathered data. It then broadcasts information of all the groups in the network. Upon receiving this information, each node finds its responsibility in the cluster by comparing its ID with the IDs of $\mathrm{CH}$. If the node is $\mathrm{CH}$, it takes the responsibility of data aggregation and data dissemination to the $\mathrm{BS}$; otherwise, the node fetches its TDMA slot and goes to sleep until its transmission turn comes. LEACH-C [14] is a centralized cluster-based routing protocol. The BS runs simulated annealing optimization to determine the optimum clusters. After cluster formation, the BS broadcasts back the cluster information in the network, and then, data dissemination starts.

K- Mean algorithm: The k-means algorithm is the most well-known partitioning method for clustering. It is the unsupervised clustering method. Grouping of date in $\mathrm{K}$ means clustering is done on their closeness to each other by Euclidean distance. Euclidean distance is used to find out which centroid is closest to each data point, and data points are assigned to the corresponding centroids. In this k- means clustering method $\mathrm{k}$ denotes the number of a cluster by the closeness of data point to the centroid which is mean of the data point of a cluster is designated to that cluster which shows minimum distance. The steps of the K-Mean algorithms are as follows:

Step 1: we arbitrarily choose k cluster centers (centroids).

Step 2: In this step, we calculate Euclidean distance then allocate every data point to the neighboring center.

Step3: now we calculate the average of all points assigned to the cluster, and then we obtained new centroid.

Step 4: we repeat step 2 and step3 until none of the cluster assignment change that means until our clusters remain stable, we repeat the algorithm. 


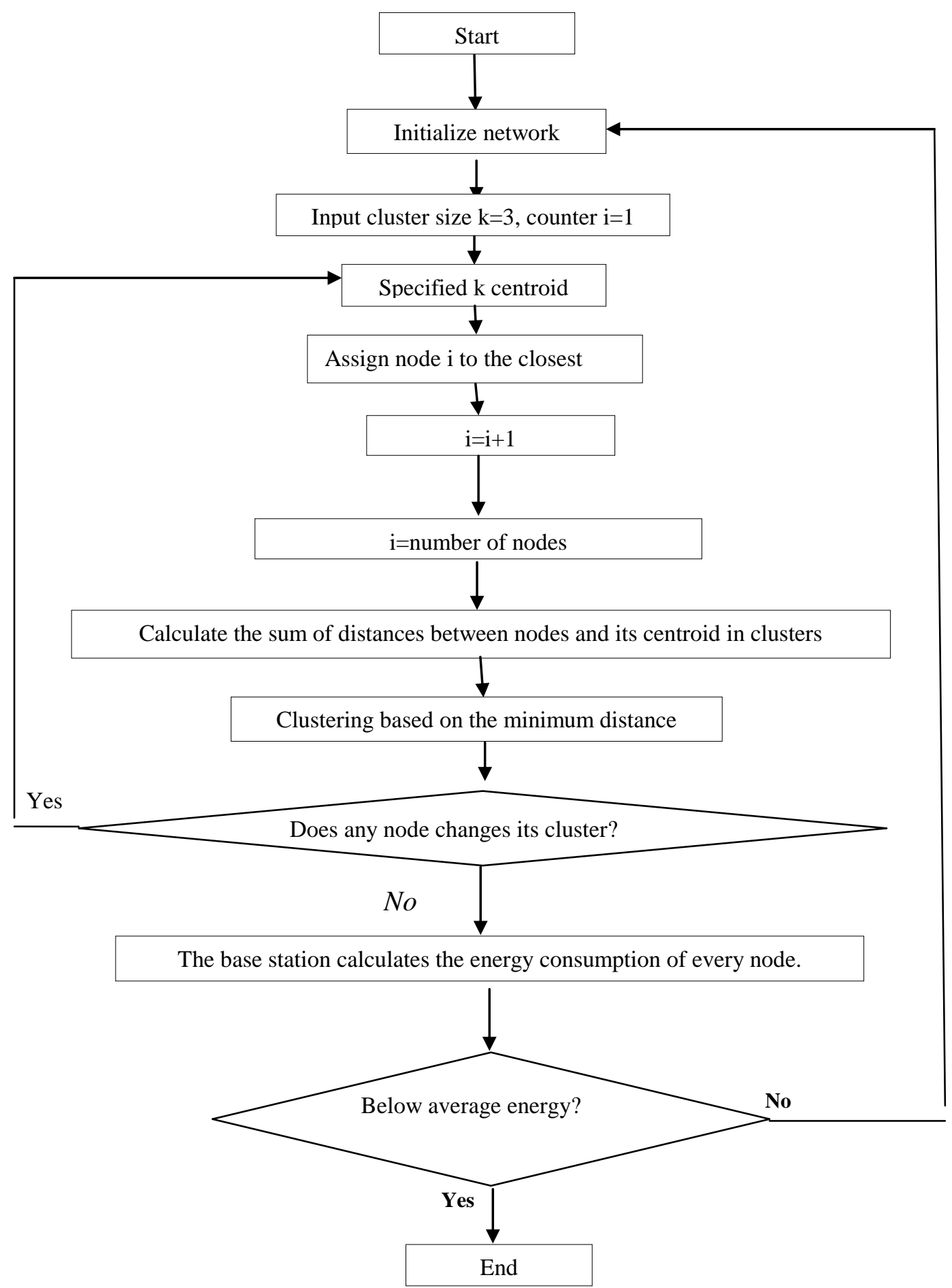

Fig.1 Flowchart of the MD- LEACH protocol 


\section{PROPOSED ALGORITHM}

In this work, we introduced an efficient cluster formation procedure using the k-means clustering algorithm called Modified-LEACH (MD-LEACH) protocol. In this algorithm, we apply K-Means partitioning method for improving the efficiency of LEACH clustering protocol. We already discussed that there are two phases in this process. First phase of communication is also called as initialization phase, in which K-means method is used to form a cluster that guarantees even groups and perfect clustering. The MDLEACH protocol is divided into several rounds, and every round contains an initialization phase and a data transmission phase. QoS parameter is improved by using the k-means method as a clustering technique and also optimized overhead during the selection of the cluster head. Flowchart of the proposed method is shown fig 1. Initially, position of the base station (BS) is stationary, some $\mathrm{k}$ groups (Clusters), i, $\mathrm{j}$ is counter, $\mathrm{n}$ indicates some nodes and the sensor field are far from the BS. Transmitting and receiving energy consumption in every transmission is equal. All the sensor nodes are homogenous in the sensor field. ModifiedLEACH (MD-LEACH) protocol is defined as follows:

Step 1: Assign $\mathrm{K}$ initial centers to arbitrarily $\mathrm{K}$ groups.

Step 2: Calculate the distance between every data points from the centers includes them in the closest center points.

$$
D=\sum_{i=1}^{k} \sum_{j=1}^{n}\left\|x_{j}-c_{i}\right\|^{2}
$$

Where, $\left\|x_{j}-c_{i}\right\|_{=}$Indicate the distance between a data point $x_{j a n d}$ the cluster center $c_{i}$

Step 3: When all aspects have been assigned, recalculate the positions of the $\mathrm{k}$ centers.

Step 4: The node has been assigned the cluster head if it has the highest energy.

Step 5: Repeat Steps 4 and 5 until convergence. This yields a separation of the points into clusters from which the metric to be limited can be determined.

Step 6: Updating the centers by the following formula

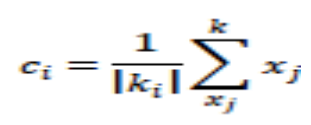

Step 7: Convergences are obtained when there is no longer variation in the cluster center and send information to BS.

\section{SIMULATION RESULTS}

In this section, we used the simulator NS2 version 2.35 to assess the performance of the proposed MD-LEACH algorithm. We also compare MD-LEACH with LEACH protocol by considering four QoS parameter namely: PDR, bandwidth, energy consumption, and throughput. In these simulations 100 nodes arbitrarily scattered in the $100 \mathrm{~m} \times 100 \mathrm{~m}$ area. All nodes have 5 joules primary energy.
The base station is located at $100 * 150$ locations. In this simulation, we used the same energy consumption model that is used in [11] and the packet size is $500 \mathrm{~kb}$.

\section{A. Packet Delivery Ratio}

It is the proportion of the number of packets received by the base station and some packets sent from the source [10]. It is clear that from Fig 2, MD-LEACH, PDR is higher than the LEACH protocol and PDR is decreased once maximize the density of nodes due to congestion in network, error rate, etc. The MD-LEACH generates a balanced number of cluster heads.

\section{B. Bandwidth}

It is the volume of data that can be transferred in a fixed amount of time. It is generally stated in bits per second (bps) or bytes per second for digital devices while it is as shown in cycles per second, or Hertz $(\mathrm{Hz})$ for analog devices. From Fig. 3 it gives better results once maximize the density of nodes while congestion of network, packet loss, and error rate affect the performance of bandwidth.

\section{Throughput}

Throughput is the proportion of effective data transfer over a communication network. From Fig.4, MDLEACH performs slightly better than the LEACH protocol while throughput can be affected by the improvement of the density of node. It is transferred large number of packets to the base station because MDLEACH generates a balanced number of cluster heads.

\section{Energy Consumption}

There are so many reasons for maximum energy consumption in the system. Some of them are the concentration of nodes, the distance of cluster head form cluster member, and distance of the BS from clusters in clustering. In Fig.5, energy consumption in MD-LEACH and LEACH is approximately the same.

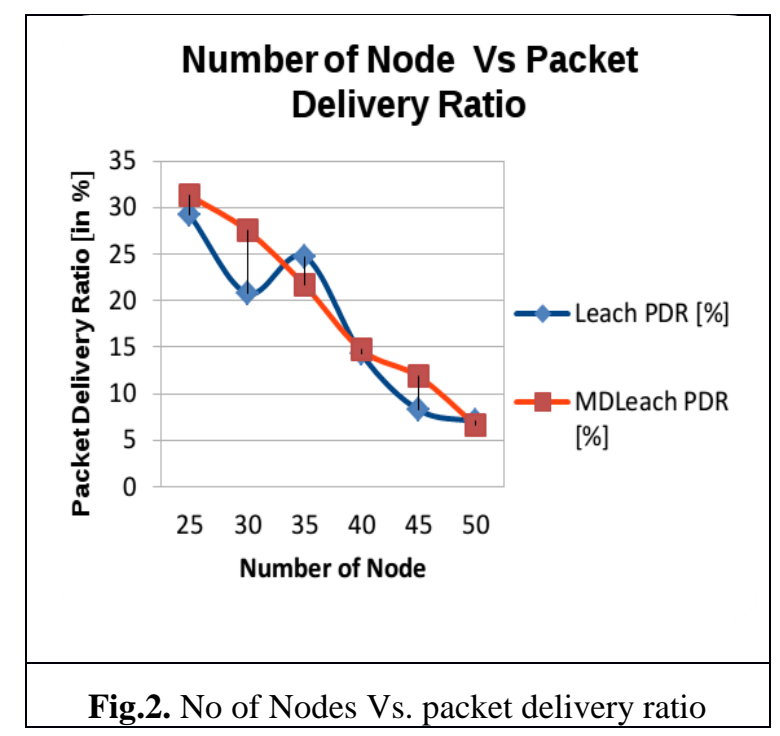

Fig.2. No of Nodes Vs. packet delivery ratio 


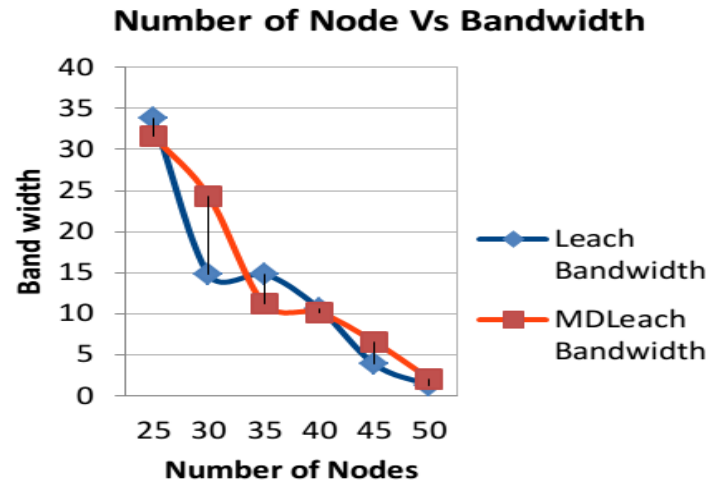

Fig.3. No of Node Vs. Bandwidth
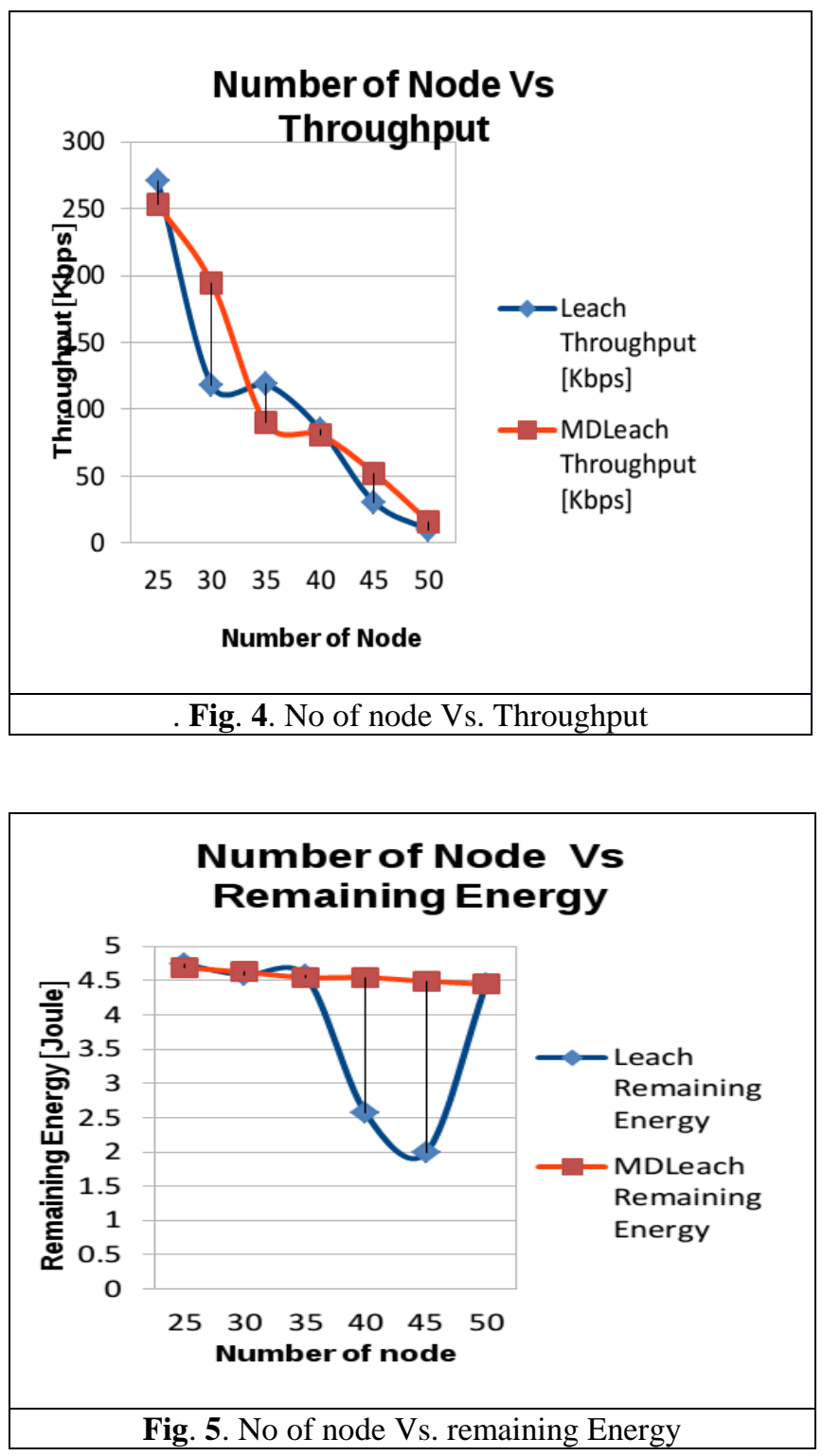

In this work, we have proposed a Modified-LEACH (MDLEACH) protocol based k-means algorithm for WSNs. The MD-LEACH protocol is a combination of previous LEACH protocol and K-means classification. There are two phases involve designing MD-LEACH protocol. In the first phase, we select the LEACH protocoling then we applied the Kmeans algorithm on the LEACH protocol. We choose the cluster by the position of centroid and cluster head by energy as well as the location of the cluster head. The obtained results show that MD-LEACH protocol performs better than LEACH protocol for all QoS parameters. It also shows that it does reduce the overhead without packet loss in data transmission from source to destination.

\section{REFERENCES}

1. Heinzelman, W.R., Chandrakasan, A., \& Balakrishnan, H., (2000). Energy-Efficient Communication Protocol for Wireless Microsensor Networks. Proceedings of the 33rd Hawaii International Conference on System Sciences, pp.

2. Sonagara, D., \& Badheka, S., (2014). Comparison of Basic Clustering Algorithms. International Journal of Computer Science and Mobile Computing, 3(10), 58-61.

3. Mechta, D., Harous, S., Alem, I., Khebbab, D., (2014). LEACH-CKM Low Energy Adaptive Clustering Hierarchy protocol with K-means and MTE," Proc.Int. Conf. INNOVATIONS, pp. 99-103.

4. Rabiaa, Elkamel, Baccar Noura, and Cherif Adnene. "Improvements in LEACH based on K-means and Gauss algorithms," Procedia Computer Science, vol.73, pp 460-467, 2015

5. Park, G. Y., Kim, H., Jeong, H. W. and Youn, H.Y. (2013). A Novel Cluster Head Selection Method based on K-Means Algorithm for Energy Efficient Wireless Sensor Network. 27th International Conference on Advanced Information Networking and Applications Workshops, pp. 910-915

6. Kaur, P., \& Bhardwaj, V. (2015). K-Means based General Self Organized Tree-Based Energy Balancing Routing Protocol for Wireless Sensor Networks. International Journal of Computer Science and Information Technologies (IJCSIT), 6(4), 3457-3464.

7. Park, G. Y., Kim, H., Jeong, H. W. \& Youn, H.Y. (2013). A Novel Cluster Head Selection Method based on K-Means Algorithm for Energy Efficient Wireless Sensor Network. 27th International Conference on Advanced Information Networking and Applications Workshops, pp. 910-915.

8. Park, G.Y., Kim, H., Jeong, H.W., \& Youn, Y.H., (2013). A Novel Cluster Head Selection Method based on K-Means Algorithm for Energy Efficient Wireless Sensor Network. 27 ${ }^{\text {th }}$ International Conference on Advanced Information Networking and Applications Workshops, pp. 910-915.

9. Bholowalia, P., \& Kumar, A., (2014). EBK-Means: A Clustering Technique based on Elbow Method and K-Means in WSN International Journal of Computer Applications. 105(9), pp. 17-24

10. Echoukairi, H., Bouragba,K., \& Ouzzif, M., (2015). Evaluation an Comparative Study Routing of Wireless Sensor Networks Hierarchical Protocols. Proceedings of the WCCS'15, pp. 235-339.

11. Heinzelman, W., Chandrakasan, A., \& Balakrishnan, H., (2002) Application-specific protocol architecture for wireless microsensor networks. IEEE Transactions on Wireless Communications, 1(4), pp 660-670.

12. Singh, S., Chand, S., \& Kumar, B., (2017). Multilevel heterogeneous network model for wireless sensor networks. Telecommunication Systems, 64(2), 259-277.

13. ZHANG, Lili et SOONG, Boon-Hee. Energy efficiency analysis of channel-aware geographic-informed forwarding (CAGIF) for wireles sensor networks. IEEE Transactions on Wireless Communications, vol. 7, no 6, p. 2033-2038, 2008. 
14. Heinzelman, W. B., (2000). Application-Specific Protocol Architectures for Wireless Networks, Microsystems Technology Laboratories, Massachusetts Institute of Technology, Cambridge, MA, USA.

15. Loscri, V., Morabito, G., \& Marano, S., (2005). A two-level hierarchy for low-energy adaptive clustering hierarchy (TL-LEACH)," in VTC2005-Fall. 2005 IEEE 62nd Vehicular Technology Conference, pp.1809-1813.

16. Singh, S., Chand, S., \& Kumar, B., (2016). Energy efficient clustering protocol using fuzzy logic for heterogeneous WSNs. Wireless Personal Communications, 86 (2), 451-475.

17. Mehmood, A., Lloret, J., Noman, M., and Song, H., (2015). Improvement of the wireless sensor network lifetime using LEACH with vice-cluster head. Adhoc \& Sensor Wireless Networks, 28(1), 117.

18. Chen, J., Li, Z., \& Kuo, Y. H., (2013). A centralized balance clustering routing protocol for a wireless sensor network," Wireless Personal Communications, 72(1), pp. 623-634.

19. Pachlor R., \& Shrimankar, D., (2017). EEHCCP: an energy-efficient hybrid clustering communication protocol for a wireless sensor network. in 9th EAI International Conference on Ad Hoc Networks, Niagara Falls, Canada, 36-41.

20. Chand, S., Singh, S. \& Kumar, B., (2014) Heterogeneous HEED Protocol for Wireless Sensor Networks. Wireless Pers Commun 77(13): 2117-2139.

21. Ye, M., Li, C., Chen, G., \& Wu, J. (2005). EECS: an energy efficient clustering scheme in wireless sensor networks. in PCCC 2005. 24th IEEE International Performance, Computing, and Communications Conference, pp. 535-540.

22. Singh, S., Chauhan, A.K., Raghav, S., Tyagi, V., \& Johari, S., (2011) Heterogeneous protocols for increasing lifetime of wireless sensor networks. Journal of Global Research in Computer Science, 2(4), 172176.

23. Singh, S., (2017). Energy efficient multilevel network model for heterogeneous WSNs. Engineering Science and Technology, an International Journal, 20(1), 105-115.

24. Singh, S., Chand, S., \& Kumar, B., (2013). 3-Tier heterogeneous network model for increasing lifetime in three dimensional WSNs. International Conference on Heterogeneous Networking for Quality, Reliability, Security and Robustness, pp. 238-247.

25. Singh, S., \& Sharma, AK., (2013). Distributed Algorithms for Maximizing Lifetime of WSNs with Heterogeneity and Adjustable Sensing Range for Different Deployment Strategies. International Journal of Information Technology and Computer Science, 5, 101-108.

\section{AUTHORS PROFILE}

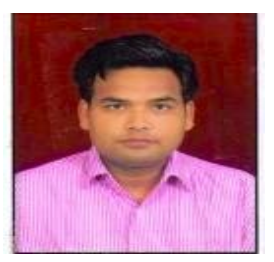

Arvinda Kushwaha received the bachelor's degree in Computer Science and Engineering, from Bundelkhand University, Jhansi, Uttar Pradesh, India in 2002 and master's degree in Software Engineering from RGPV Bhopal, Madhya Pradesh, India in 2012. Currently he is pursuing his Ph.D. degree in computer engineering at Jamia Millia Islamia, Delhi, India. He has published more than 15 research papers in reputed international journals including Thomson Reuters (SCI \& Scopus) and conferences including IEEE and it's also available online His research interests include, wireless sensor networks, and cloud computing.

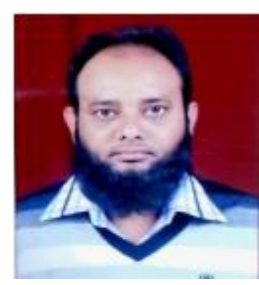

Dr. Mohd. Amjad is currently working as Associate Professor in the Department of Computer Engineering, F/o Engineering \& Technology, Jamia Millia Islamia (Central University), New Delhi. He received B.Tech. Degree from A.M.U. Aligarh in computer Engineering, M.Tech. Degree in Information Technology from GGSIP University New Delhi and Ph.D. from Jamia Millia Islamia, from the Department of Computer Engineering New Delhi. He has published more than 50 research papers in reputed international journals including Thomson Reuters (SCI \& Scopus) and conferences including IEEE and it's also available online. His research interest includes Network Security, Internet and mobile computing, Mobile Ad hoc Networks and wireless sensor networks. Dr. Amjad has more than 16 Years of teaching experience at U.G and P.G. Level. 